\title{
Prognostic value and biological significance of GUCY1A2 in gastric cancer: a bioinformatics analysis
}

\section{Shan Huang}

The Seventh Affiliated Hospital, Sun Yat-sen University

Min Wei

The Seventh Affiliated Hospital, Sun Yat-sen University

Mengyuan Zhu

The Seventh Affiliated Hospital, Sun Yat-sen University

Xiaofen Pan ( $\square$ panxf8@mail.sysu.edu.cn )

The Seventh Affiliated Hospital, Sun Yat-sen University

\section{Research Article}

Keywords: GUCY1A2, gastric cancer, prognostic biomarker.

Posted Date: March 17th, 2021

DOI: https://doi.org/10.21203/rs.3.rs-274107/v1

License: (c) (i) This work is licensed under a Creative Commons Attribution 4.0 International License.

Read Full License 


\section{Abstract}

Background: Guanylate cyclase 1 soluble subunit alpha 2 (sGCa2), also known as GUCY1A2, was reported to be upregulated and promoted tumorigenesis in some cancers. But whether GUCY1A2 was abnormally expressed and the prognostic value in gastric cancer was unknown. The current study aimed to find out the prognostic value of GUCY1A2 in gastric cancer by analyzing data from The Cancer Genome Atlas (TCGA). Methods: Wilcoxon signed-rank test, cox regression analysis and multivariant analysis were used to analyze the relationship between clinical characteristic and GUCY1A2 expression level. Kaplan-Meier method was used to analyze the association of GUCY1A2 and overall survival. Gene set enrichment analysis (GSEA) was used to identify GUCY1A2-related signaling pathway.

Results: Compared to normal tissue, expression of GUCY1A2 was significantly increased in gastric cancer $(p=0.000)$. Increased GUCY1A2 was associated with advance T stage $(p=0.012)$ and poor survival $(p=0.022)$. Univariate analysis showed that high GUCY1A2 expression was associated with a poor overall survival (HR:1.44, 95\% confidence interval [Cl]: 1.03-2.02, $p=0.03$ ). Multivariate analysis indicated that GUCY1A3 remained an independent prognostic predictor of overall survival (HR:1.75, 95\% confidence interval $[\mathrm{CI}]: 1.20-2.56, p=0.00)$. GSEA revealed that calcium signaling pathway, MAPK signaling pathway, TGF- $\beta$ signaling pathway and Wnt signaling pathway were enriched in GUCY1A2 high expression phenotype.

Conclusions: GUCY1A2 maybe a potential prognostic predictor of poor survival in gastric cancer. Calcium signaling pathway, MAPK signaling pathway, TGF- $\beta$ signaling pathway and Wnt signaling pathway may be the key signaling pathway regulated by GUCY1A2.

\section{Introduction}

Gastric cancer is one of the most common malignant tumors in the world, with the fifth highest incidence rate and the third highest mortality rate(1). The incidence rate of gastric cancer shows geographical differences. It is most frequently occurred in East Asia, South America and Eastern Europe and less common seen in Western Europe, Australia and North America (2). More than 95\% of gastric cancers are classified as adenocarcinomas (3). Helicobacter pylori infection is the most important cause of gastric cancer(4). Other risk factors include smoking, alcohol consumption, high salt intake, et al(5-7). The only curative therapy for gastric cancer is surgical resection(8). However, about $50 \%$ of patients presents with advanced diseases at diagnosis and about $40-60 \%$ of patients relapsed after surgical resection(9). The standard treatment of these patients is systemic chemotherapy and target therapy(10). However, prognosis of metastasis gastric cancer is poor, with the 5-year overall survival (OS) rate of $5-20 \%(11)$. Searching for new prognostic biomarkers and treatment targets may help to improve the prognosis of gastric cancer.

The cyclic GMP (cGMP)/protein Kinase G (PKG) pathway participates in the endogenous apoptotic process in several types of cancers(12-14). cGMP, which is one of the most important intracellular 
signaling molecule in mammal cells, is generated from GTP by two classes of guanylate cyclases (GCs): soluble GC (sGC) and particulate GC (pGC)(15). Guanylate cyclase 1 soluble subunit alpha 1 (sGCa1) and alpha 2 (sGCa2) are subunits of sGCs. It is reported that sGCa1 is increased in prostate cancer and overexpression of sGCa1 is related to chemoresistance(16). Another research showed that sGCa1 promoted cell proliferation, migration and survival of cervical cancer cells(17). Inhibition of basal activity of sGC/cGMP pathway induced p53-dependent apoptosis in ovarian cancer cells. Guanylate cyclase soluble subunit alpha-2, also known as GUCY1A2, is found to be upregulated in cervical cancer and correlated with superficial tumor growth(18). However, the expression level and prognostic value of GUCY1A2 in gastric cancer remains unknown.

Thus, the current study aimed at evaluating the expression and prognostic value of GUCY1A2 in gastric cancer by bioinformatics analysis. In addition, we performed the gene set enrichment analysis (GSEA) to study the biological pathway related to the GUCY1 regulation. The data used in this study was obtained from TCGA database (https://portal.gdc.cancer.gov/).

\section{Methods}

\subsection{TCGA data download and bioinformatic analysis}

The gene expression data and corresponding clinical data of gastric cancer (project ID: TCGA-STAD) were downloaded from TCGA database (https://portal.gdc.cancer.gov/). Expression data of GUCY1A2 was extracted. Then the expression difference of GUCY1A2 between normal and tumor samples were compared. Clinical information was extracted and patients with incomplete clinical information were excluded. Finally, there were 349 patients were included into cox regression and multivariate cox analysis. The media expression value of GUCY1A2 were calculated. Patients with GUCY1A2 expression level higher than media value were distributed to the high expression group and others were distributed to the low expression group. Overall survival in high expression and low expression group were compared. Cox regression and multivariate cox analysis were used to analysis the influence of clinical characteristics on the survival.

\subsection{Gene set enrichment analysis}

Software GSEA_4.1.0 was used to perform gene set enrichment analysis (GSEA). The expression matrix file and phenotype file were prepared before GSEA. GUCY1A2 expression level was used as a phenotype label. Gene set permutations number was set to 1000 . Then the expression matrix file and phenotype file were imported and GSEA was performed.

\subsection{Statistical analysis}

Statistical analyses were conducted by $R$ software $(R \times 64$ 3.6.2). The comparison of GUCY1A2 expression between normal and tumor sample was analyzed by Wilcoxon signed-rank test. Clinical characteristics associated to survival was analyzed by Cox regression and Kaplan-Meier method. To 
further compare the effect of GUCY1A2 expression on overall survival along with other clinical factors, multivariate Cox analysis was performed.

\section{Results}

\subsection{Patient characteristics}

Totally 349 cases with clinical data were downloaded from TCGA in January 2021. 136 (39.0\%) cases were male. 47 (13.5\%) patients were with stage I disease, 110 (31.5\%) patients were with stage II disease, $155(44.4 \%)$ patients were with stage III disease and 37 (10.6\%) patients were with stage IV disease. T1, T2, T3, T4 diseases were found in 17 (4.9\%), 72 (20.6\%), 168 (48.1\%), 92 (26.4\%) patients, respectively. $67.9 \%$ of the patients had lymph node invasion. Of which, 91 cases were N1 disease, 75 cases were N2 disease, 71 cases were N3 disease. 24(6.9\%) patients had distant metastases. Clinical characteristics of patients were shown in table 1.

Table 1. Patient characteristics of gastric cancer

\begin{tabular}{llll}
\hline Characteristic & & N(total=349) & \% \\
\hline Age & $\leq 65$ & 160 & 45.8 \\
Gender & 665 & 189 & 54.2 \\
& male & 136 & 39.0 \\
Grade & female & 213 & 61.0 \\
& G1 & 6 & 1.7 \\
Clinical stage & I & 47 & 34.4 \\
& G2 & 120 & 63.9 \\
& II & 110 & 13.5 \\
T stage & III & 155 & 31.5 \\
& IV & 37 & 44.4 \\
& 1 & 17 & 40.6 \\
& 2 & 72 & 20.6 \\
N stage & 3 & 168 & 48.1 \\
& 4 & 92 & 26.4 \\
& 0 & 112 & 32.1 \\
M stage & 1 & 91 & 26.1 \\
& 2 & 75 & 21.5 \\
& 3 & 71 & 20.3 \\
& 0 & 325 & 93.1 \\
& 1 & 24 & 6.9
\end{tabular}




\subsection{GUCY1A2 expression was increased in gastric carcinoma patients}

Data of 343 gastric carcinoma samples and 30 normal samples with GUCY1A2 expression information were downloaded from TCGA database in January 2021. GUCY1A2 expression level were compared in normal samples and tumors samples. In comparison to normal samples, GUCY1A2 expression was significantly increased in tumor samples (Fig. 1A and 1B).

\subsection{Increased GUCY1A2 was associated with poor overall survival}

Patients with complete survival information and GUCY1A2 expression information were included in survival analysis. As shown in Fig. 2A, patients with high GUCY1A2 expression showed a worse prognosis than patients with low GUCY1A2 expression. We also analyzed the association between GUCY1A2 expression and other clinical characteristics. Results showed that expression level of GUCY1A2 was correlated to histological grade, $\mathrm{T}$ stage. Patients who with higher histological grade and T stage had higher GUCY1A2 level. But GUCY1A2 expression was independent of age, gender, clinical stage, lymph node invasion or distant metastasis (Fig. 2B-2H).

\subsection{GUCY1A2 was an independent prognostic factor in gastric carcinoma}

Univariate cox regression analysis showed that age, stage, distant metastasis and high GUCY1A2 expression level was correlated significantly with poor survival. HR was 1.44 and $95 \%$ confidence intervals was1.03-2.02 ( $p=0.03$ ) (Tab. 2). Age, gender, clinical stage and increased GUCY1A2 expression level remained associated with survival at multivariate analysis (Tab. 3 and Fig. 3 ).

Table 2. Association of clinical characteristics and survival by univariate cox regression

\begin{tabular}{lcc}
\hline Characteristic & $\mathrm{HR}(95 \% \mathrm{CI})$ & $\mathrm{p}$ value \\
\hline Age & $1.03(1.01-1.05)$ & $0.004^{*}$ \\
Gender & $1.50(0.98-2.30)$ & 0.062 \\
Grade & $1.25(0.85-1.84)$ & 0.252 \\
Clinical stage & $1.51(1.20-1.91)$ & $0.001^{*}$ \\
T stage & $1.28(1.00-1.63)$ & 0.050 \\
M stage & $2.07(1.07-3.98)$ & $0.030^{*}$ \\
N stage & $1.54(0.97-2.45)$ & 0.070 \\
GUCY1A2 & $1.44(1.03-2.02)$ & $0.034^{*}$ \\
\hline
\end{tabular}

HR: Hazard Ratio, CI: confident interval 
Table 3. Association of clinical characteristics and survival by Multivariate analysis

\begin{tabular}{lcc}
\hline Characteristic & HR(95\%CI) & p value \\
\hline Age & $1.05(1.02-1.07)$ & $0.000^{*}$ \\
Gender & $1.57(1.01-2.44)$ & $0.045^{*}$ \\
Grade & $1.33(0.89-1.99)$ & 0.167 \\
Clinical stage & $1.71(1.09-2.69)$ & $0.019^{*}$ \\
T stage & $1.03(0.73-1.45)$ & 0.086 \\
M stage & $1.83(0.80-4.16)$ & $0.150^{*}$ \\
N stage & $0.69(0.34-1.38)$ & 0.292 \\
GUCY1A2 & $1.76(1.20-2.56)$ & $0.004^{*}$ \\
\hline
\end{tabular}

HR: Hazard Ratio, CI: confident interval

\subsection{GUCY1A2 related signaling pathways identified by GSEA}

We performed GSEA to found out signaling pathways which are differentially activated between low GUCY1A2 expression and high GUCY1A2 expression data sets in gastric carcinoma. Differences in enrichment of KEGG pathways (c2.cp.kegg.v7.2.symbols) were analyzed. The enriched pathways were selected according to the normalized enrichment score (NES) (Tab.4). Pathways of adherens junction, calcium signaling pathway, cell adhesion molecules cams, ECM receptor interaction, focal adhesion, MAPK signaling pathway, TGF- $\beta$ signaling pathway, Wnt signaling pathway were enriched in GUCY1A2 high expression group (Fig. 4).

Table 4. Gene set enriched in HIST1H1D high phenotype 


\begin{tabular}{lcl}
\hline Gene Set Name & NES & NOM p-val \\
\hline KEGG_CALCIUM_SIGNALING_PATHWAY & 2.08 & 0.000 \\
KEGG_FOCAL_ADHESION & 2.06 & 0.002 \\
KEGG_ECM_RECEPTOR_INTERACTION & 2.23 & 0.000 \\
KEGG_TGF_BETA_SIGNALING_PATHWAY & 1.80 & 0.016 \\
KEGG_MAPK_SIGNALING_PATHWAY & 1.76 & 0.008 \\
KEGG_ADHERENS_JUNCTION & 1.68 & 0.032 \\
KEGG_CELL_ADHESION_MOLECULES_CAMS & 1.65 & 0.048 \\
KEGG_WNT_SIGNALING_PATHWAY & 1.59 & 0.038 \\
\hline
\end{tabular}

NES: Normalized enrichment score; NOM: Nominal. Gene sets with NOM p-val<0.05 were considered as significant.

\section{Discussion}

Soluble guanylate cyclase (sGC) is a catalytic enzyme that catalyzing the conversion of guanosine $5^{\prime}-$ triphosphate (GTP) into cyclic guanosine $3^{\prime}, 5^{\prime}$-monophosphate (cGMP). sGC is one of the main receptors of nitric oxide (NO). When SGC binds to NO, the catalytic activity was increased by 100 - to 200 -folds. Thus it plays an important role in the NO-cGMP pathway(19). It has been shown that NO/sGC/cGMP pathway plays an important role in tumor development. Increased cGMP leads to increased protein kinase G (PKG) activity and promotes prostate cancer cell proliferation(20). Inhibition of NO/cGMP/PKG pathway results in suppression of migration and invasion in breast cancer cells(21).

sGC a $150 \mathrm{kDa}$ heterodimer consisting of two subunits, subunit $a$ and subunit $\beta(22)$. Both of the subunits are required for catalytic activity(23). There are two isoforms of both subunits $₫ a 1, a 2$ and $\beta 1 \otimes \beta 2 . a 1 / \beta 1$ is the most abundant form. But there is indistinguishable activity between the $\alpha 1 / \beta 1$ and $a 2 / \beta 1$ heterodimers(24). It has been shown that sGC subunit $\alpha 1$ and $\alpha 2$, also known as GUCY1A1 and GUCY1A2, is increased in some type of cancers and promotes cell proliferation, migration and survival of cancer cells(16-18). But whether GUCY1A2 was abnormally expressed and the prognostic value in gastric cancer was unknown. 
In the current study, we found that GUCY1A2 expression was increased in gastric cancer in comparison to normal tissues. Increased GUCY1A2 expression was associated with advance T stage and poor prognosis in gastric cancer. GSEA indicated that adherens junction, calcium signaling pathway, cell adhesion molecules cams, ECM receptor interaction, focal adhesion, MAPK signaling pathway, TGF- $\beta$ signaling pathway, Wnt signaling pathway was enriched in GUCY1A2 high expression phenotype. Results suggested that high level of GUCY1A2 may serve as a predictive biomarker of poor prognosis in gastric cancer.

Some research has indicated that upregulation of the calcium signaling pathway, Wnt signaling pathway, MAPK signaling pathway and TGF- $\beta$ signaling pathway were associated with tumor development and progression(25-28). A recent study showed that increased cGMP resulted in increased tumor cell stemness and promoted metastasis in breast cancer by activating the cGMP-dependent PKG and MAPK signaling pathway(29). Activation of SGC and increased generation of cGMP also induced activation of TGF- $\beta 1(30)$. However, little is known about how SGC subunit GUCY1A2 was related to the above signaling pathway and further exploration is needed.

\section{Conclusion}

Our current research showed that GUCY1A2 mays serve as a potential prognostic biomarker of poor survival in gastric cancer. Calcium signaling pathway, MAPK signaling pathway, TGF- $\beta$ signaling pathway and Wnt signaling pathway maybe the critical pathway regulated by GUCY1A2 in gastric cancer. However, GUCY1A2 expression level in this research was mRNA expression rather than protein expression and using mRNA to predict protein expression was not perfect. Further study is needed to validate the results and prove the biological influence of GUCY1A2.

\section{List Of Abbreviations}

TCGA, The Cancer Genome Atlas

GSEA, gene set enrichment analysis

KEGG, Kyoto Encyclopedia of Genes and Genomes

GUCY1A2, guanylate cyclase soluble subunit alpha-2

sGC, soluble Guanylate cyclase

OS, overall survival

cGMP, cyclic guanosine monophosphate

PKG, protein kinase $G$ 
TGF- $\beta$, transforming growth factor $\beta$

MAPK, mitogen activated protein kinase

\section{Declarations}

\section{Ethics approval and consent to participate}

Not applicable

\section{Consent for publication}

Not applicable

\section{Availability of data and materials}

The datasets generated and analysed during the current study are available in the TCGA repository, http://cancergenome.nih.gov/.

\section{Competing interests}

The authors declare that they have no competing interests

\section{Funding}

National Natural Science Foundation of Guangdong, China(2020A1515110923).

\section{Authors' contributions}

SH, MW, MZ and XP had full access to all the data in the study and take responsibility for the integrity of the data and the accuracy of the data analysis;

Study concept and design: XP,

Acquisition of data: $\mathrm{SH}, \mathrm{MW}, \mathrm{MZ}$

Analysis and interpretation of data: $\mathrm{SH}$ and $\mathrm{MW}$

Drafting of the manuscript: $S H, M W, M Z, X P$

Statistical analysis: SH and MW

Supervision: $\mathrm{MZ}$ and $\mathrm{XP}$

\section{Acknowledgements}

Not applicable. 


\section{References}

1. Bray F, Ferlay J, Soerjomataram I, Siegel RL, Torre LA, Jemal A. Global cancer statistics 2018: GLOBOCAN estimates of incidence and mortality worldwide for 36 cancers in 185 countries. CA: a cancer journal for clinicians. 2018;68(6):394-424.

2. Collaborators GBDSC. The global, regional, and national burden of stomach cancer in 195 countries, 1990-2017: a systematic analysis for the Global Burden of Disease study 2017. The lancet Gastroenterology \& hepatology. 2020;5(1):42-54.

3. Ma J, Shen H, Kapesa L, Zeng S. Lauren classification and individualized chemotherapy in gastric cancer. Oncology letters. 2016;11(5):2959-64.

4. Bornschein J, Selgrad M, Warnecke M, Kuester D, Wex T, Malfertheiner P. H. pylori infection is a key risk factor for proximal gastric cancer. Digestive diseases and sciences. 2010;55(11):3124-31.

5. Karimi P, Islami F, Anandasabapathy S, Freedman ND, Kamangar F. Gastric cancer: descriptive epidemiology, risk factors, screening, and prevention. Cancer epidemiology, biomarkers \& prevention : a publication of the American Association for Cancer Research, cosponsored by the American Society of Preventive Oncology. 2014;23(5):700-13.

6. Yusefi AR, Bagheri Lankarani K, Bastani P, Radinmanesh M, Kavosi Z. Risk Factors for Gastric Cancer: A Systematic Review. Asian Pacific journal of cancer prevention : APJCP. 2018;19(3):591603.

7. Zhao JK, Wu M, Kim CH, Jin ZY, Zhou JY, Han RQ, et al. Jiangsu Four Cancers Study: a large casecontrol study of lung, liver, stomach, and esophageal cancers in Jiangsu Province, China. European journal of cancer prevention : the official journal of the European Cancer Prevention Organisation. 2017;26(4):357-64.

8. Lutz MP, Zalcberg JR, Ducreux M, Ajani JA, Allum W, Aust D, et al. Highlights of the EORTC St. Gallen International Expert Consensus on the primary therapy of gastric, gastroesophageal and oesophageal cancer - differential treatment strategies for subtypes of early gastroesophageal cancer. European journal of cancer. 2012;48(16):2941-53.

9. Biagioni A, Skalamera I, Peri S, Schiavone N, Cianchi F, Giommoni E, et al. Update on gastric cancer treatments and gene therapies. Cancer metastasis reviews. 2019;38(3):537-48.

10. Smyth EC, Verheij M, Allum W, Cunningham D, Cervantes A, Arnold D, et al. Gastric cancer: ESMO Clinical Practice Guidelines for diagnosis, treatment and follow-up. Annals of oncology : official journal of the European Society for Medical Oncology. 2016;27(suppl 5):v38-v49.

11. Wagner AD, Syn, N.L., Moehler, M., Grothe, W., Yong, W.P., Tai,B.-C., Ho, J., Unverzagt, S., . Chemotherapy for advanced gastric cancer. . Cochrane Database Syst Rev 8, CD004064 h. 2017.

12. Fallahian F, Karami-Tehrani F, Salami S, Aghaei M. Cyclic GMP induced apoptosis via protein kinase $\mathrm{G}$ in oestrogen receptor-positive and -negative breast cancer cell lines. The FEBS journal. 2011;278(18):3360-9. 
13. Fajardo AM, Piazza GA, Tinsley HN. The role of cyclic nucleotide signaling pathways in cancer: targets for prevention and treatment. Cancers. 2014;6(1):436-58.

14. Browning DD, Kwon IK, Wang R. cGMP-dependent protein kinases as potential targets for colon cancer prevention and treatment. Future medicinal chemistry. 2010;2(1):65-80.

15. Gileadi 0 . Structures of soluble guanylate cyclase: implications for regulatory mechanisms and drug development. Biochemical Society transactions. 2014;42(1):108-13.

16. Cai C, Hsieh CL, Gao S, Kannan A, Bhansali M, Govardhan K, et al. Soluble guanylyl cyclase alpha1 and p53 cytoplasmic sequestration and down-regulation in prostate cancer. Molecular endocrinology. 2012;26(2):292-307.

17. Ronchetti SA, Pino MTL, Cordeiro G, Bollani SN, Ricci AG, Duvilanski BH, et al. Soluble guanylyl cyclase alpha1 subunit is a key mediator of proliferation, survival, and migration in ECC-1 and HeLa cell lines. Scientific reports. 2019;9(1):14797.

18. Eggen T, Sager G, Arnes M, Pettersen I, Orbo A. Expression of iNOS-a favourable prognostic marker for early-stage carcinoma of the uterine cervix. Anticancer research. 2011;31(6):2319-25.

19. Childers KC, Garcin ED. Structure/function of the soluble guanylyl cyclase catalytic domain. Nitric oxide : biology and chemistry. 2018;77:53-64.

20. Zhou F, Gao S, Han D, Han W, Chen S, Patalano S, et al. TMPRSS2-ERG activates NO-cGMP signaling in prostate cancer cells. Oncogene. 2019;38(22):4397-411.

21. Schwappacher R, Rangaswami H, Su-Yuo J, Hassad A, Spitler R, Casteel DE. cGMP-dependent protein kinase lbeta regulates breast cancer cell migration and invasion via interaction with the actin/myosin-associated protein caldesmon. Journal of cell science. 2013;126(Pt 7):1626-36.

22. Russwurm M, Behrends $S$, Harteneck $C$, Koesling D. Functional properties of a naturally occurring isoform of soluble guanylyl cyclase. The Biochemical journal. 1998;335 (Pt 1):125-30.

23. Harteneck C, Wedel B, Koesling D, Malkewitz J, Bohme E, Schultz G. Molecular cloning and expression of a new alpha-subunit of soluble guanylyl cyclase. Interchangeability of the alphasubunits of the enzyme. FEBS letters. 1991;292(1-2):217-22.

24. Nakane M. Soluble guanylyl cyclase: physiological role as an NO receptor and the potential molecular target for therapeutic application. Clinical chemistry and laboratory medicine. 2003;41(7):865-70.

25. Li X, Wei R, Wang M, Ma L, Zhang Z, Chen L, et al. MGP Promotes Colon Cancer Proliferation by Activating the NF-kappaB Pathway through Upregulation of the Calcium Signaling Pathway. Molecular therapy oncolytics. 2020;17:371-83.

26. Taciak B, Pruszynska I, Kiraga L, Bialasek M, Krol M. Wnt signaling pathway in development and cancer. Journal of physiology and pharmacology : an official journal of the Polish Physiological Society. 2018;69(2).

27. Colak S, Ten Dijke P. Targeting TGF-beta Signaling in Cancer. Trends in cancer. 2017;3(1):56-71. 
28. Fang JY, Richardson BC. The MAPK signalling pathways and colorectal cancer. The Lancet Oncology. 2005;6(5):322-7.

29. Lv Y, Wang X, Li X, Xu G, Bai Y, Wu J, et al. Nucleotide de novo synthesis increases breast cancer stemness and metastasis via cGMP-PKG-MAPK signaling pathway. PLoS biology. 2020;18(11):e3000872.

30. Metukuri MR, Namas R, Gladstone C, Clermont T, Jefferson B, Barclay D, et al. Activation of latent transforming growth factor-beta1 by nitric oxide in macrophages: role of soluble guanylate cyclase and MAP kinases. Wound repair and regeneration : official publication of the Wound Healing Society [and] the European Tissue Repair Society. 2009;17(4):578-88.

\section{Figures}




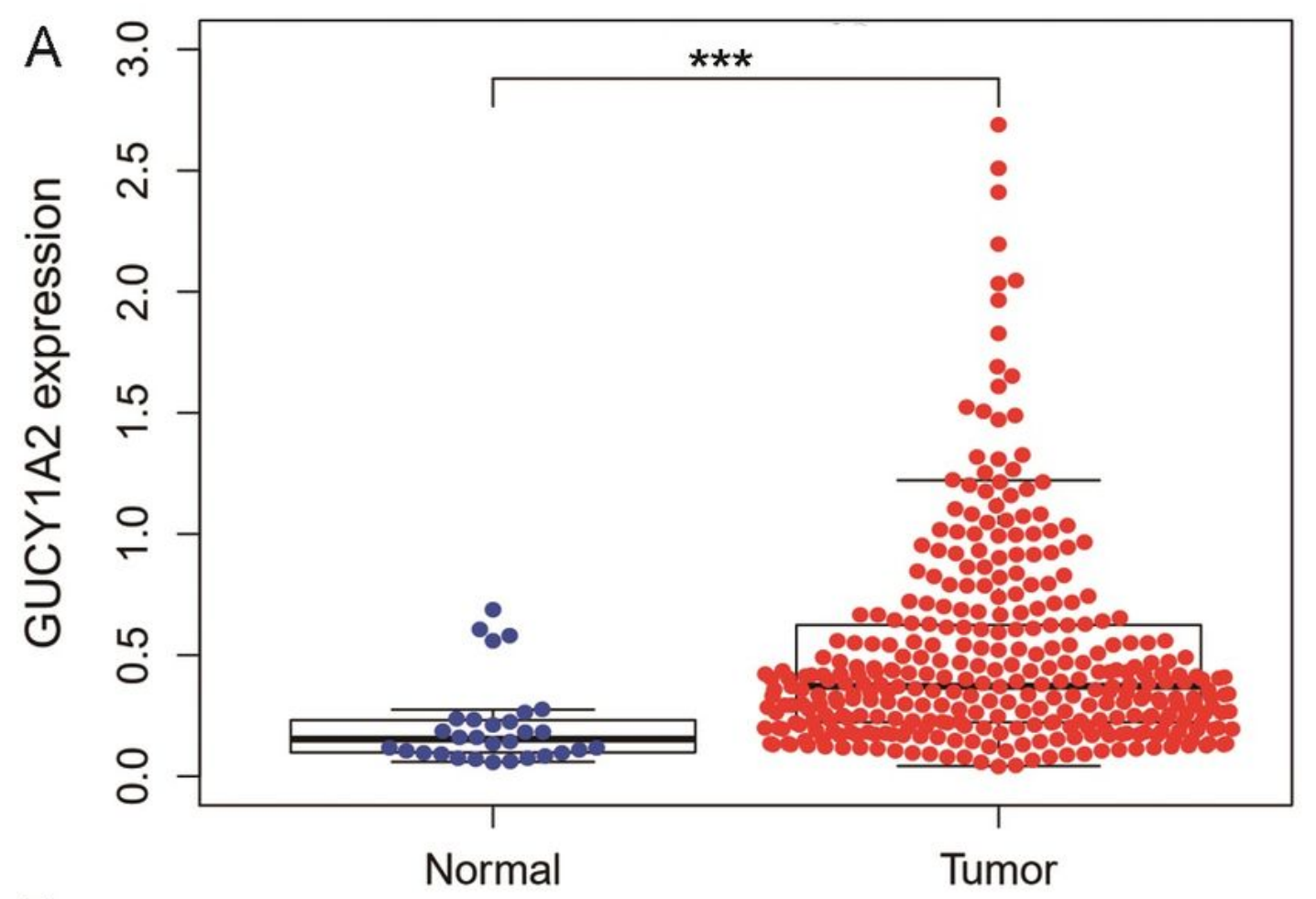

B

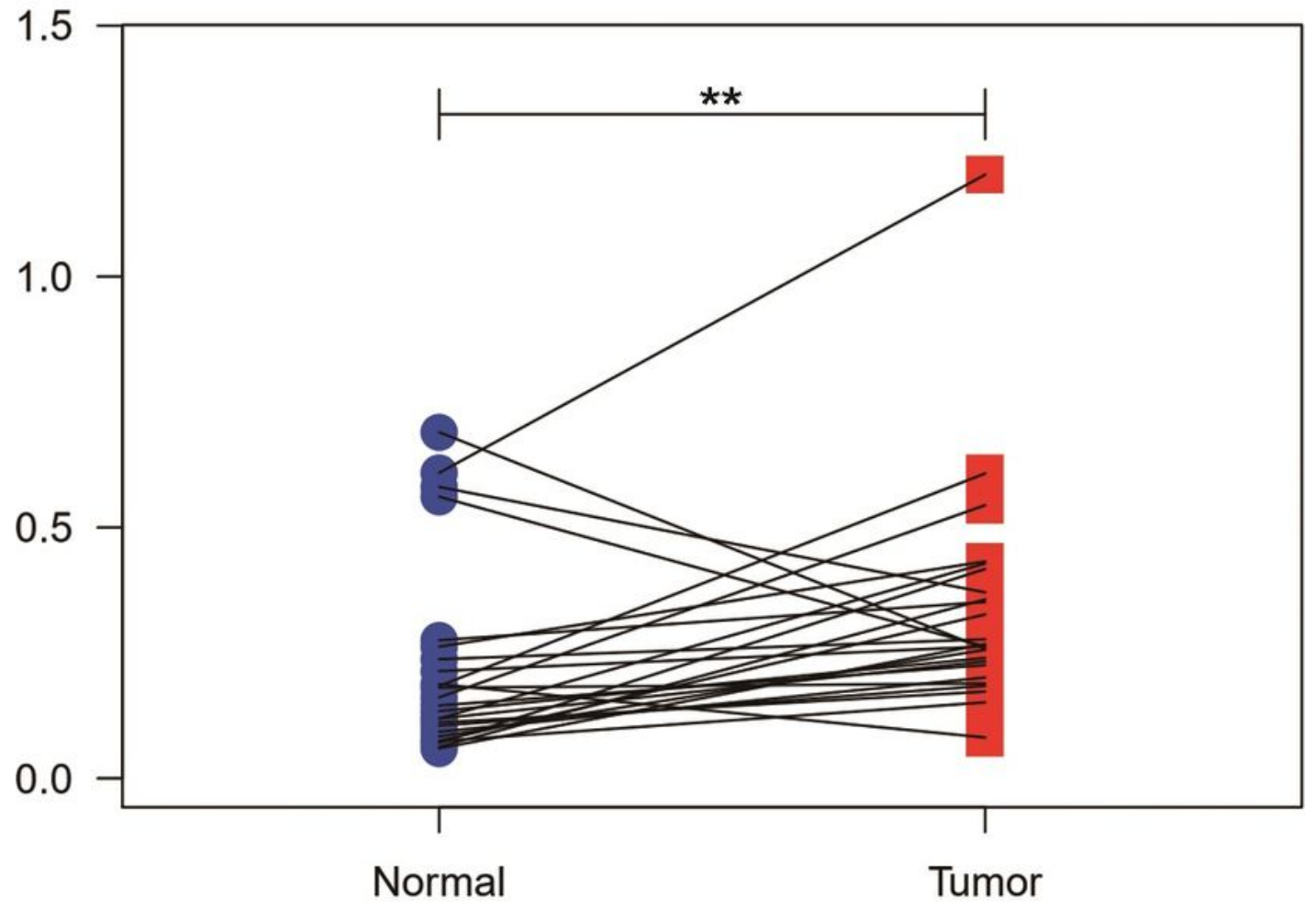

Figure 1

(A) GUCY1A2 expression in gastric cancer was significantly higher than normal samples (B) GUCY1A2 expression in cancer sample was also higher than normal sample from the same patient. ${ }^{\star \star} p<0.01$, $* \star * p<0.001$ 

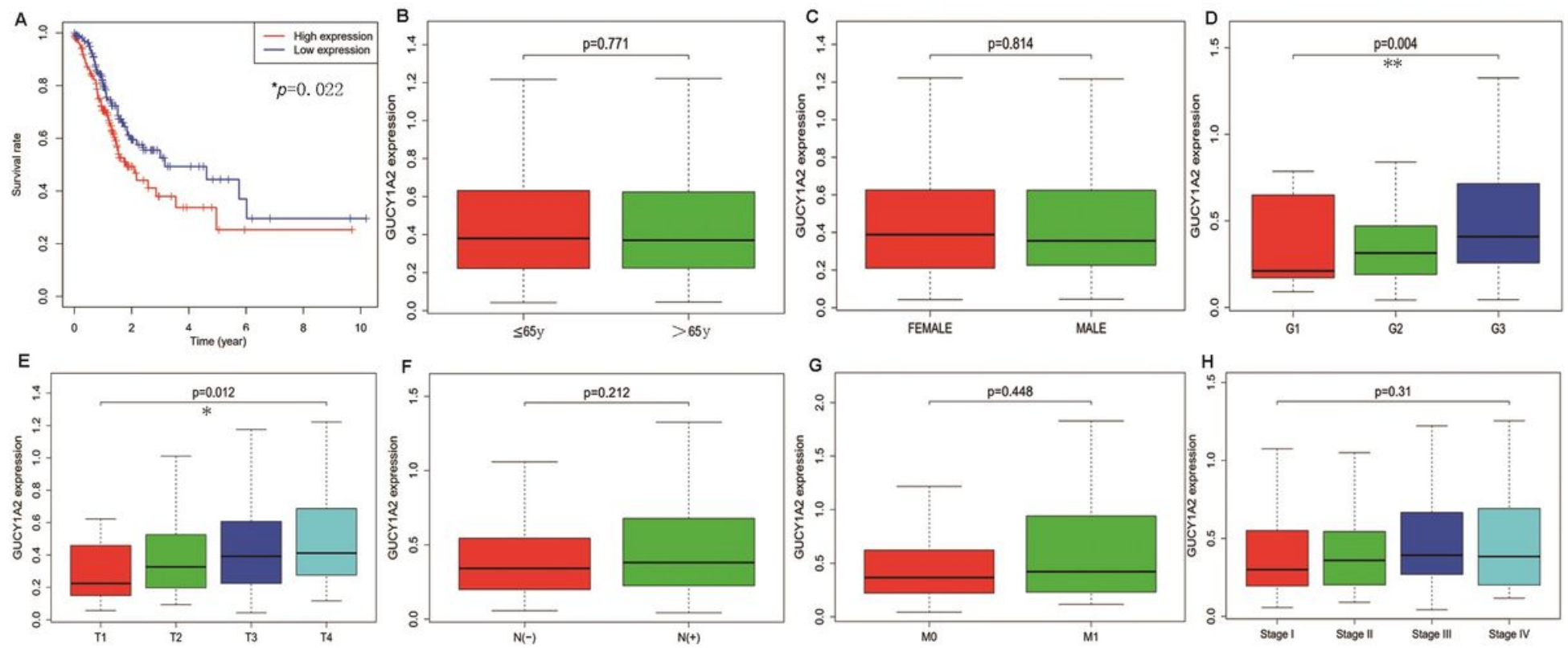

Figure 2

The correlation of GUCY1A2 expression with survival and other clinical characteristics. (A) Patients with increased GUCY1A2 expression showed worse survival. (B) Correlation of GUCY1A2 expression and age. (C) Correlation of GUCY1A2 expression and gender. (D) Correlation of GUCY1A2 expression and histological grade. (E) Correlation of GUCY1A2 expression and T stage. (F) Correlation of GUCY1A2 expression and lymph nodes metastasis. (G) Correlation of GUCY1A2 expression and distant metastasis. $(H)$ Correlation of GUCY1A2 expression and clinical stage. ${ }^{*} p<0.05,{ }^{\star \star} p<0.01,{ }^{\star \star \star} p<0.001$ 


\section{Hazard ratio}

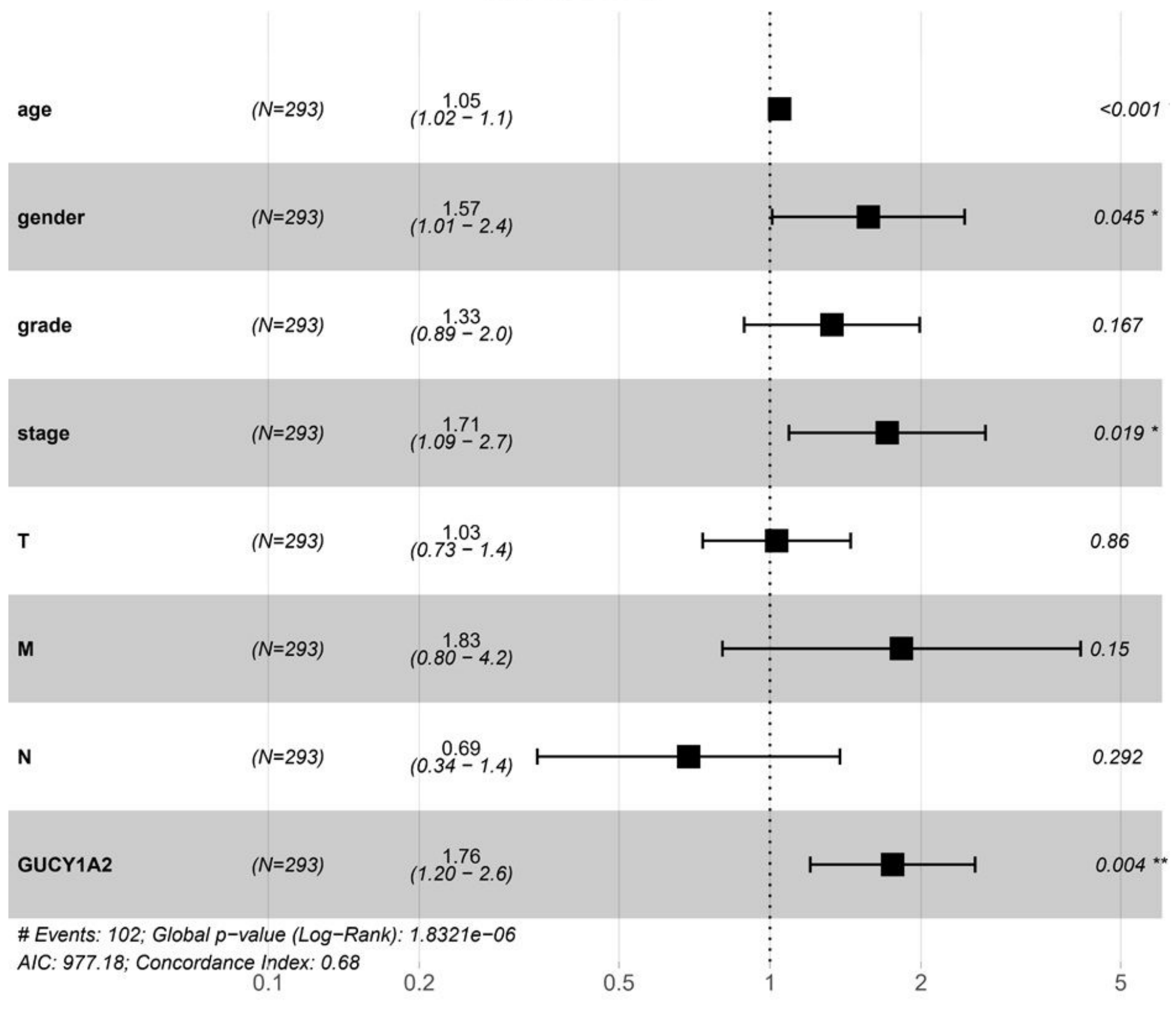

\section{Figure 3}

Multivariate analysis showed that age, gender, clinical stage and high expression of GUCY1A2 was independent predictors of poor survival. ${ }^{*} \mathrm{p}<0.05,{ }^{*} \mathrm{p}<0.01$ 
A

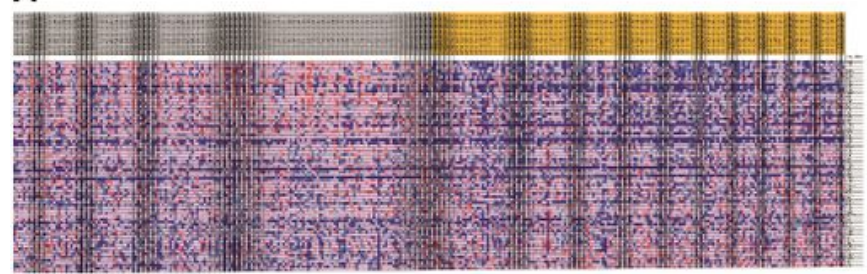

C

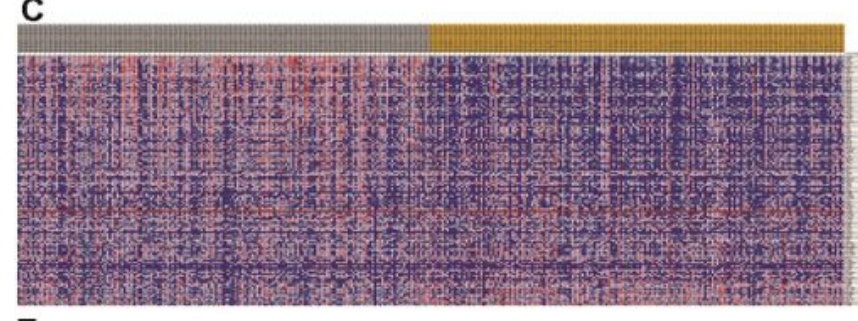

E

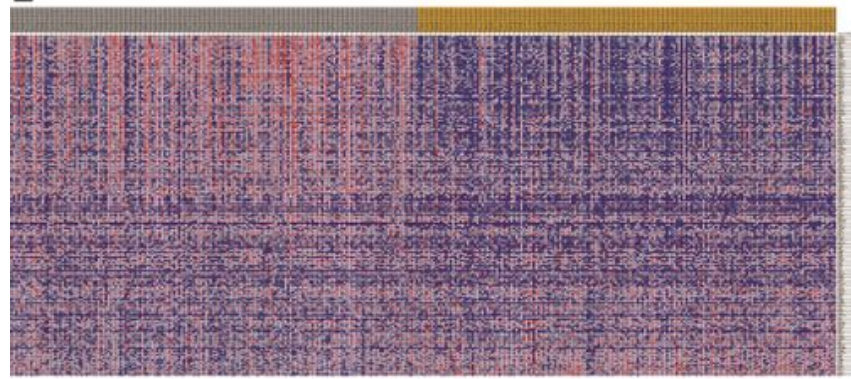

G

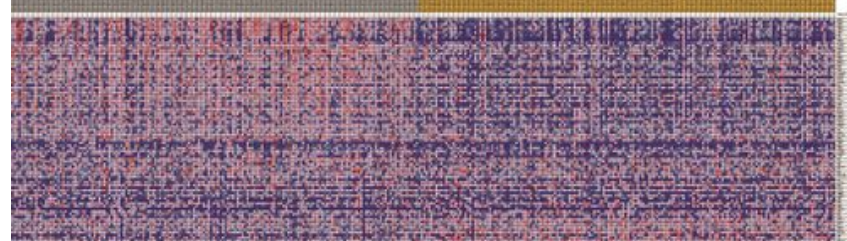

B

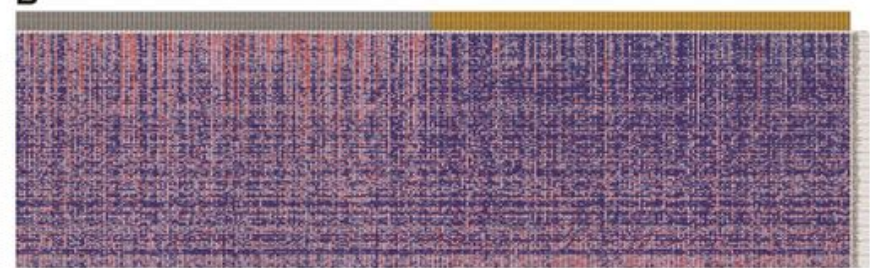

D

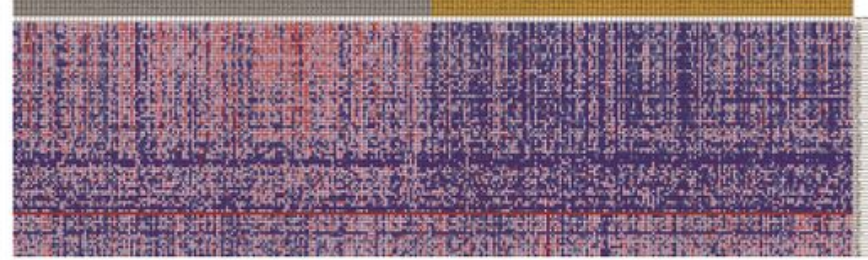

$\mathbf{F}$

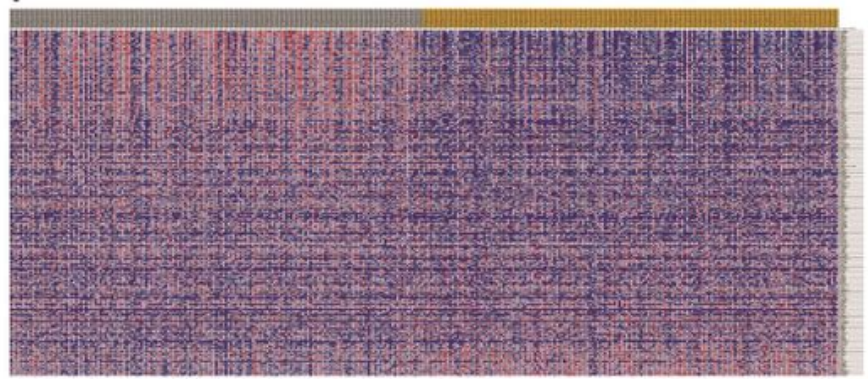

H

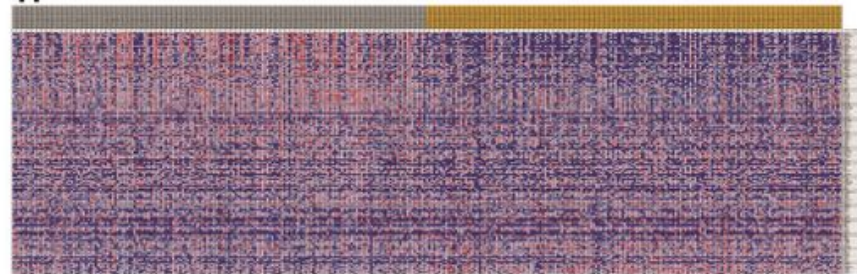

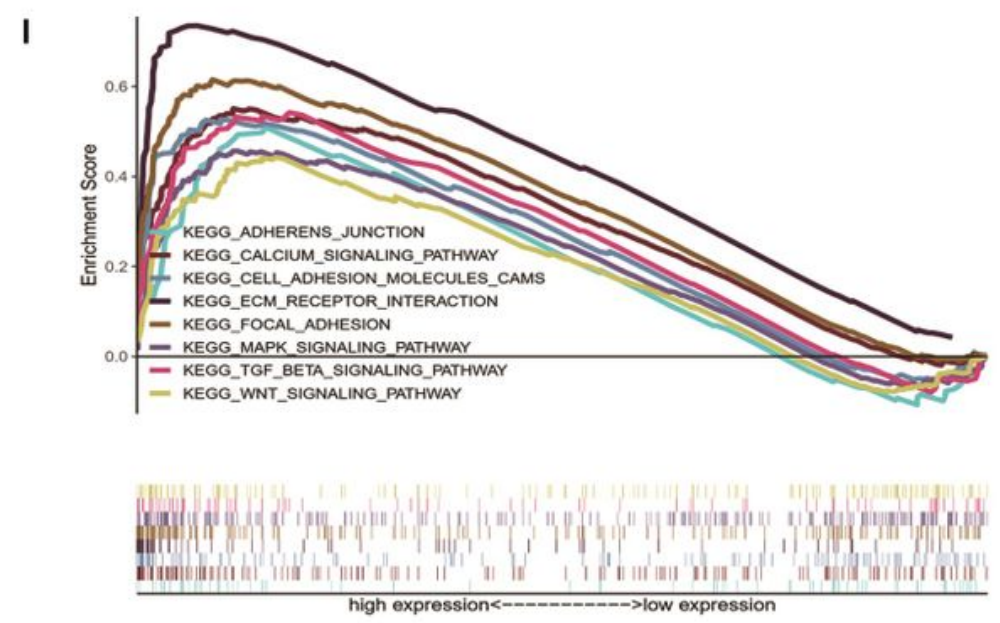

\section{Figure 4}

Results of gene set enrichment analysis showed that adherens junction, calcium signaling pathway, cell adhesion molecules cams, ECM receptor interaction, focal adhesion, MAPK signaling pathway, TGF- $\beta$ signaling pathway, Wnt signaling pathway were enriched in GUCY1A2 high expression group. (A) KEGG adherens junction pathway. (B) KEGG calcium signaling pathway. (C)KEGG cell adhesion molecules cams. (D) KEGG ECM receptor interaction pathway. (E)KEGG focal adhesion pathway. (F)KEGG MAPK 
signaling pathway. (G)TGF- $\beta$ signaling pathway. (H)Wnt signaling pathway. (I) Gene set enrichment analysis result of the above pathways. 